\title{
Supersymmetric solutions of matter-coupled 7D $N=2$ gauged supergravity
}

\author{
Parinya Karndumri* and Patharadanai Nuchino \\ String Theory and Supergravity Group, Department of Physics, Faculty of Science, \\ Chulalongkorn University, 254 Phayathai Road, Pathumwan, Bangkok 10330, Thailand
}

(Received 27 July 2018; published 10 October 2018)

\begin{abstract}
We study supersymmetric solutions within seven-dimensional $N=2$ gauged supergravity coupled to three vector multiplets in seven dimensions. The gauged supergravity contains six vector fields that gauge the $S O(4) \sim S O(3) \times S O(3)$ symmetry and admits two $N=2$ supersymmetric $\mathrm{AdS}_{7}$ vacua with $S O(4)$ and $S O(3)_{\text {diag }} \subset S O(3) \times S O(3)$ symmetries. We consider solutions interpolating between two asymptotically locally $\mathrm{AdS}_{7}$ geometries in the presence of a three-form field. For a particular value of the two $S O(3)$ gauge coupling constants, the $S O(3)_{\text {diag }}$ supersymmetric $\mathrm{AdS}_{7}$ vacuum does not exist, but the solutions can be uplifted to 11 dimensions by a known reduction ansatz. We also study solutions of this type and their embedding in M theory. We further extend these solutions to include the $S O(3)_{\text {diag }}$ gauge fields and argue that, in general, this generalization does not lead to supersymmetric solutions.
\end{abstract}

DOI: 10.1103/PhysRevD.98.086012

\section{INTRODUCTION}

Over the past 20 years, the AdS/CFT correspondence has been widely tested and confirmed by a large number of interesting results. It has been applied to holographic studies of strongly coupled field theories in various space-time dimensions. One of the interesting cases is $\mathrm{AdS}_{7} / \mathrm{CFT}_{6}$ correspondence which has been argued to describe the dynamics of M5 branes in M theory since the first proposal of the correspondence in Ref. [1].

As in other cases, $\mathrm{AdS}_{7} / \mathrm{CFT}_{6}$ correspondence can be efficiently investigated by using gauged supergravities in seven dimensions. For example, $\mathrm{AdS}_{7} \times S^{4}$ geometry of M theory, dual to $N=(2,0)$ superconformal field theory (SCFT) in six dimensions, can be described by $S O(5)$ $N=4$ gauged supergravity [2,3]. In this paper, we are interested in the case of half-maximal $N=2$ gauged supergravity. The corresponding $\mathrm{AdS}_{7}$ vacua are dual to $N=(1,0)$ SCFTs; see, e.g., $[4,5]$. Some of these SCFTs can be obtained from an orbifold of the $N=(2,0)$ SCFTs [6,7], and, recently, interest in $N=(1,0)$ SCFTs has been increased by many new results; see [8-12] for an incomplete list.

$N=2$ gauged supergravity has been constructed for a long time in Refs. [13-15]. These theories, however, do not

\footnotetext{
parinya.ka@hotmail.com

danai.nuchino@hotmail.com
}

Published by the American Physical Society under the terms of the Creative Commons Attribution 4.0 International license. Further distribution of this work must maintain attribution to the author(s) and the published article's title, journal citation, and DOI. Funded by SCOAP . admit any $\mathrm{AdS}_{7}$ vacua. The existence of an $\mathrm{AdS}_{7}$ vacuum requires an additional deformation in the form of a mass term for the three-form field, dual to the two-form field in the $N=2$ gravity multiplet. The $N=2$ gauged supergravity including both types of deformations has been given in Ref. [16]; see also [17]. An extension of this $N=2$ gauged supergravity to include vector multiplets has been given in Ref. [18]. A number of supersymmetric $\mathrm{AdS}_{7}$ vacua and various types of holographic solutions within this gauged supergravity have been studied in Refs. [19-21]. A classification of possible gauge groups that can give rise to maximally supersymmetric $A d S_{7}$ vacua has also been given in Ref. [22]. Most of the previously known solutions of pure and matter-coupled $N=2$ gauged supergravity involve only the metric and scalar fields, although the results of Refs. [20,21] do include solutions with nonvanishing gauge fields.

Supersymmetric solutions of pure $N=2$ gauged supergravity with all bosonic fields, including the three-form and gauge fields, nonvanishing have appeared recently in Ref. [23] along with the embedding in M theory by using the result of Ref. [24]. The solution without the $S U(2)$ gauge fields has also been uplifted to massive type-IIA theory in Ref. [25], in which the solution is interpreted as a two-dimensional conformal defect in $N=(1,0)$ SCFT. In the present work, we are interested in similar solutions in $N=2$ gauged supergravity coupled to three vector multiplets with the $S O(4) \sim S O(3) \times S O(3)$ gauge group. In this case, the maximally supersymmetric $\mathrm{AdS}_{7}$ vacuum is dual to an $N=(1,0)$ SCFT with flavor symmetry $S O(3)$. The solutions presented here should give an extension of the results in Refs. [19,23] and represent more general solutions of $N=2$ seven-dimensional gauged supergravity. 
The paper is organized as follow. In Sec. II, we give a short review of the matter-coupled $N=2$ gauged supergravity in seven dimensions. The aim of this section is to give relevant formulas which will be used throughout the paper. Supersymmetric solutions interpolating between two asymptotically locally $\mathrm{AdS}_{7}$ geometries and solutions flowing from an asymptotically locally $\mathrm{AdS}_{7}$ with $S O(4)$ symmetry to a singular geometry are given in Sec. III. These solutions are obtained by using the $\mathrm{AdS}_{3} \times S^{3}$-sliced domain wall ansatz. We also discuss the embedding of the latter type of solutions in 11-dimensional supergravity. In Sec. IV, we study similar solutions with nonvanishing $S O(3)_{\text {diag }}$ gauge fields and argue that this does not give rise to supersymmetric solutions. Some conclusions and comments on the results are given in Sec. V. All bosonic field equations of $N=2$ gauged supergravity coupled to vector multiplets are given in Appendix A. A consistent reduction ansatz for special values of the gauge coupling constants is reviewed in Appendix B.

\section{II. $N=2$ GAUGED SUPERGRAVITY IN SEVEN DIMENSIONS}

We first give a brief review of $N=2$ gauged supergravity in seven dimensions with a topological mass term. All of the conventions and notations are essentially the same as those in Ref. [18], to which the reader is referred for more detail.

The half-maximal $N=2$ supergravity in seven dimensions can couple to an arbitrary number $n$ of vector multiplets, the only matter multiplets in $N=2$ supersymmetry. The field contents are given, respectively, by

$$
\begin{array}{ll}
\text { supergravity multiplet : } & \left(e_{\mu}^{\hat{\mu}}, \psi_{\mu}^{A}, A_{\mu}^{i}, \chi^{A}, B_{\mu \nu}, \sigma\right), \\
\text { vector multiplet : } & \left(A_{\mu}, \lambda^{A}, \phi^{i}\right)^{r}
\end{array}
$$

Curved and flat space-time indices are denoted by $\mu, \nu, \ldots$. and $\hat{\mu}, \hat{\nu}, \ldots$, respectively. $B_{\mu \nu}$ and $\sigma$ are the two-form and the dilaton fields, respectively. The two-form field will be dualized to a three-form field $C_{\mu \nu \rho}$, which admits a topological mass term leading to a massive deformation of the $N=2$ supergravity. Indices $i, j=1,2,3$ label triplets of $S U(2)_{R} \sim S O(3)_{R}$ symmetry. The dilaton $\sigma$ can be described by a coset space $S O(1,1) \sim \mathbb{R}^{+}$.

Each of the vector multiplets, labeled by indices $r$, $s=1,2, \ldots, n$, consists of a vector field $A_{\mu}$, two gaugini $\lambda^{A}$, and three scalars $\phi^{i}$. Indices $A, B, \ldots=1,2$ label a doublet of the $S U(2)_{R}$ symmetry and will be generally suppressed throughout this paper. There are $3 n$ scalar fields $\phi^{i r}$ parametrizing the $S O(3, n) / S O(3) \times S O(n)$ coset manifold. These can be efficiently described by a coset representative of the form

$$
L=\left(L_{I}{ }^{i}, L_{I}{ }^{r}\right), \quad I=1, \ldots, 3+n .
$$

The inverse of $L$ will be denoted by

$$
L^{-1}=\left(L_{i}^{I}, L_{r}^{I}\right)
$$

Since $L$ is an element of $S O(3, n)$, we have the following relations:

$$
\begin{aligned}
& \eta_{I J}=-L_{I}{ }^{i} L_{J}{ }^{i}+L_{I}{ }^{r} L_{J}{ }^{r}, \\
& L^{I}{ }_{i}=\eta^{I J} L_{J i}, \quad \text { and } \quad L_{r}^{I}=\eta^{I J} L_{J} .
\end{aligned}
$$

It should be noted that indices $i, j$ and $r, s$ are raised and lowered by $\delta_{i j}$ and $\delta_{r s}$, respectively, while the full $S O(3, n)$ indices $I, J$ are raised and lowered by the $S O(3, n)$ invariant tensor $\eta_{I J}=\operatorname{diag}(---+\cdots+)$. With these conventions, relations involving components of $L$ can be written as

$$
\begin{gathered}
L_{I}{ }^{i}=L_{I i}, \quad L_{I}^{r}=L_{I r}, \\
L_{I}{ }^{i} L^{I}{ }_{j}=-\delta_{j}^{i}, \quad L_{I}^{r} L^{I}{ }_{s}=\delta_{s}^{r} .
\end{gathered}
$$

Gaugings of $N=2$ supergravity can be obtained by promoting a subgroup $G_{0}$ of the global symmetry group $\mathbb{R}^{+} \times S O(3, n)$ to be a local symmetry. If the gauging does not involve the $\mathbb{R}^{+}$factor, the embedding of $G_{0}$ in $S O(3, n)$ is described by the $S O(3, n)$ tensor $f_{I J}{ }^{K}$ identified with the structure constants of the gauge group $G_{0}$ via the gauge algebra

$$
\left[T_{I}, T_{J}\right]=f_{I J}{ }^{K} T_{K},
$$

where $T_{I}$ denote the gauge generators. In the embedding tensor formalism, $f_{I J}{ }^{K}$ is a component of the full embedding tensor; see [26] for more detail.

For the gauging to be a consistent one, preserving all of the original supersymmetry, $f_{I J}{ }^{K}$ must satisfy the conditions

$$
f_{I J K}=\eta_{K L} f_{I J}^{L}=f_{[I J K]} \quad \text { and } \quad f_{[I J}^{L} f_{K] L}^{M}=0 .
$$

Apart from the gauging, there is also a massive deformation given by adding a topological mass term to the three-form field $C_{\mu \nu \rho}$. This additional deformation is crucial for the gauged supergravity to admit $\mathrm{AdS}_{7}$ vacua.

The bosonic Lagrangian including both the gauging and the massive deformation can be written as

$$
\begin{aligned}
L= & \frac{1}{2} R * \mathbb{I}-\frac{1}{2} e^{\sigma} a_{I J} * F_{(2)}^{I} \wedge F_{(2)}^{J}-\frac{1}{2} e^{-2 \sigma} * H_{(4)} \wedge H_{(4)} \\
& -\frac{5}{8} * d \sigma \wedge d \sigma-\frac{1}{2} * P^{i r} \wedge P_{i r}+\frac{1}{\sqrt{2}} H_{(4)} \wedge \omega_{(3)} \\
& -4 h H_{(4)} \wedge C_{(3)}-V * \mathbb{I},
\end{aligned}
$$

where we have used the form language for convenience in dealing with the field equations. The constant $h$ describes the topological mass term for the three-form $C_{(3)}$ with $H_{(4)}=d C_{(3)}$. 
The scalar potential is given by

$$
V=\frac{1}{4} e^{-\sigma}\left(C^{i r} C_{i r}-\frac{1}{9} C^{2}\right)+16 h^{2} e^{4 \sigma}-\frac{4 \sqrt{2}}{3} h e^{\frac{3 \sigma}{2}} C,
$$

where $C$ and $C_{i r}$ are defined in term of the coset representative as, respectively,

$$
\begin{aligned}
C & =-\frac{1}{\sqrt{2}} f_{I J}{ }^{K} L^{I}{ }_{i} L^{J}{ }_{j} L_{K k} \epsilon^{i j k}, \\
C_{i r} & =\frac{1}{\sqrt{2}} f_{I J}{ }^{K} L^{I}{ }_{j} L^{J}{ }_{k} L_{K r} \epsilon^{i j k} .
\end{aligned}
$$

The scalar kinetic term is written in term of the vielbein $P_{\mu}^{i r}$ on the $S O(3, n) / S O(3) \times S O(n)$ coset defined by

$$
P_{\mu}^{i r}=L^{I r}\left(\delta_{I}^{K} \partial_{\mu}+f_{I J}^{K} A_{\mu}^{J}\right) L_{K}^{i} .
$$

The scalar matrix $a_{I J}$ appearing in the kinetic term of vector fields is given by

$$
a_{I J}=L_{I}{ }^{i} L_{J i}+L_{I}^{r} L_{J r}
$$

Finally, the Chern-Simons three-form satisfying $d \omega_{(3)}=$ $F_{(2)}^{I} \wedge F_{(2)}^{I}$ is defined by

$$
\omega_{(3)}=F_{(2)}^{I} \wedge A_{(1)}^{I}-\frac{1}{6} f_{I J}^{K} A_{(1)}^{I} \wedge A_{(1)}^{J} \wedge A_{(1) K}
$$

with the gauge field strength tensors $F_{(2)}^{I}=d A_{(1)}^{I}+$ $\frac{1}{2} f_{J K}^{I} A_{(1)}^{J} \wedge A_{(1)}^{K}$. The associated bosonic field equations are collected in Appendix A. The gauge coupling constants are included in $f_{I J}{ }^{K}$.

Other ingredients which are relevant for finding supersymmetric solutions are supersymmetry transformations of fermions. With all fermionic fields vanishing, these are given by

$$
\begin{aligned}
\delta \psi_{\mu}= & 2 D_{\mu} \epsilon-\frac{\sqrt{2}}{30} e^{-\frac{\sigma}{2}} C \gamma_{\mu} \epsilon \\
& -\frac{1}{240 \sqrt{2}} e^{-\sigma} H_{\rho \sigma \lambda \tau}\left(\gamma_{\mu} \gamma^{\rho \sigma \lambda \tau}+5 \gamma^{\rho \sigma \lambda \tau} \gamma_{\mu}\right) \epsilon \\
& -\frac{i}{20} e^{\frac{\sigma}{2}} F_{\rho \sigma}^{i} \sigma^{i}\left(3 \gamma_{\mu} \gamma^{\rho \sigma}-5 \gamma^{\rho \sigma} \gamma_{\mu}\right) \epsilon-\frac{4}{5} h e^{2 \sigma} \gamma_{\mu} \epsilon, \quad(14) \\
\delta \chi= & -\frac{1}{2} \gamma^{\mu} \partial_{\mu} \sigma \epsilon-\frac{i}{10} e^{\frac{\sigma}{2}} F_{\mu \nu}^{i} \sigma^{i} \gamma^{\mu \nu} \epsilon-\frac{1}{60 \sqrt{2}} e^{-\sigma} H_{\mu \nu \rho \sigma} \gamma^{\mu \nu \rho \sigma} \epsilon \\
+ & \frac{\sqrt{2}}{30} e^{-\frac{\sigma}{2}} C \epsilon-\frac{16}{5} e^{2 \sigma} h \epsilon, \\
\delta \lambda^{r} & =i \gamma^{\mu} P_{\mu}^{i r} \sigma^{i} \epsilon-\frac{1}{2} e^{\frac{\sigma}{2}} F_{\mu \nu}^{r} \gamma^{\mu \nu} \epsilon-\frac{i}{\sqrt{2}} e^{-\frac{\sigma}{2}} C^{i r} \sigma^{i} \epsilon,
\end{aligned}
$$

where $\sigma^{i}$ are the usual Pauli matrices. The covariant derivative of $\epsilon$ is given by

$$
D_{\mu} \epsilon=\partial_{\mu} \epsilon+\frac{1}{4} \omega_{\mu}^{a b} \gamma_{a b} \epsilon+\frac{1}{2 \sqrt{2}} Q_{\mu}^{i} \sigma^{i} \epsilon
$$

where $Q_{\mu}^{i}=\frac{i}{\sqrt{2}} \epsilon^{i j k} Q_{\mu}^{j k}$ is defined in terms of the composite connection

$$
Q_{\mu}^{i j}=L^{I j}\left(\delta_{I}^{K} \partial_{\mu}+f_{I J}^{K} A_{\mu}^{J}\right) L_{K}^{i}
$$

\section{III. $\mathrm{AdS}_{3} \times S^{3}$-SLICED DOMAIN WALLS WITH THE THREE-FORM FIELD}

In this section, we will study supersymmetric solutions involving the seven-dimensional metric, scalars, and the three-form field. We will consider the case of $n=3$ vector multiplets and the $S O(4) \sim S O(3) \times S O(3)$ gauge group. The first $S O(3)$ factor is identified with the $S O(3)_{R} \sim$ $S U(2)_{R} R$ symmetry. The corresponding structure constants are given by

$$
f_{I J K}=\left(g_{1} \epsilon_{i j k}, g_{2} \epsilon_{r s t}\right) .
$$

For a particular case of $g_{2}=g_{1}$, the resulting gauged supergravity can be embedded in 11-dimensional supergravity [27].

An explicit parametrization of the $S O(3,3) / S O(3) \times$ $S O(3)$ coset can be achieved by defining $366 \times 6$ matrices of the form

$$
\left(e_{I J}\right)_{K L}=\delta_{I K} \delta_{J L}, \quad I, J \ldots=1, \ldots, 6 .
$$

Noncompact generators of $S O(3,3)$ are accordingly given by

$$
Y_{i r}=e_{i, r+3}+e_{r+3, i}, \quad i, r=1, \ldots, 3 .
$$

We first truncate all of the nine scalars in $S O(3,3) / S O(3) \times S O(3)$ coset to scalars which are singlet under $S O(3)_{\text {diag }} \subset S O(3) \times S O(3)$. There is only one singlet scalar corresponding to the noncompact generator (see [19] for more detail):

$$
Y_{s}=Y_{11}+Y_{22}+Y_{33} .
$$

The coset representative can be written as

$$
L=e^{\phi Y_{s}}
$$

The scalar potential is straightforwardly computed to be 


$$
\begin{aligned}
V= & \frac{1}{32} e^{-\sigma}\left[\left(g_{1}^{2}+g_{2}^{2}\right)[\cosh (6 \phi)-9 \cosh (2 \phi)]\right. \\
& -8 g_{1} g_{2} \sinh ^{3}(2 \phi) \\
& \left.+8\left[g_{2}^{2}-g_{1}^{2}+64 h^{2} e^{5 \sigma}+32 e^{\frac{5 \sigma}{2}} h\left(g_{1} \cosh ^{3} \phi-g_{2} \sinh ^{3} \phi\right)\right]\right] .
\end{aligned}
$$

There are two supersymmetric $\mathrm{AdS}_{7}$ critical points for this potential.

(i) $\mathrm{AdS}_{7}$ with $S O(4)$ symmetry:

$$
\sigma=\phi=0, \quad V_{0}=-240 h^{2} .
$$

(ii) $\mathrm{AdS}_{7}$ with $S O(3)_{\text {diag }}$ symmetry:

$$
\begin{aligned}
\sigma & =-\frac{1}{5} \ln \left[\frac{g_{2}^{2}-256 h^{2}}{g_{2}^{2}}\right], \quad \phi=\frac{1}{2} \ln \left[\frac{g_{2}-16 h}{g_{2}+16 h}\right], \\
V_{0} & =-\frac{240 g_{2}^{\frac{8}{5}} h^{2}}{\left(g_{2}^{2}-256 h^{2}\right)^{\frac{4}{5}}} .
\end{aligned}
$$

We have set $g_{1}=-16 h$ in order to make the dilaton $\sigma$ vanish at the $S O(4)$ critical point. This is equivalent to a redefinition of $\sigma$ by an appropriate shift. $V_{0}$ is the value of the scalar potential at the critical point. Holographic renormalization group flow solutions interpolating between these two critical points and flows to nonconformal field theories have already been given in Ref. [19].

\section{A. Solutions flowing between $\operatorname{AdS}_{7}$ vacua}

In this paper, we generalize the solutions studied in Ref. [19] by including a nonvanishing three-form field in the solutions. Following Ref. [23], we take the metric ansatz to be an $\mathrm{AdS}_{3} \times S^{3}$-sliced domain wall

$$
d s^{2}=e^{2 U(r)} d s_{\mathrm{AdS}_{3}}^{2}+e^{2 V(r)} d r^{2}+e^{2 W(r)} d s_{S^{3}}^{2}
$$

with the metrics on $\mathrm{AdS}_{3}$ and $S^{3}$ given by

$$
\begin{aligned}
d s_{\mathrm{AdS}_{3}}^{2} & =\frac{1}{\tau^{2}}\left[\left(d x^{1}\right)^{2}+\cosh ^{2} x^{1}\left(d x^{2}\right)^{2}-\left(d t-\sinh x^{1} d x^{2}\right)^{2}\right], \\
d s_{S^{3}}^{2} & =\frac{1}{\kappa^{2}}\left[\left(d \theta_{2}\right)^{2}+\cos ^{2} \theta_{2}\left(d \theta_{3}\right)^{2}+\left(d \theta_{1}+\sin \theta_{2} d \theta_{3}\right)^{2}\right] .
\end{aligned}
$$

The seven-dimensional coordinates are taken to be $x^{\mu}=$ $\left(x^{a}, r, x^{m}\right)$ with $a=0,1,2$ and $m=4,5,6$. We will also use $x^{0}=t$ and $x^{3}=r$ in the following analysis. The corresponding flat indices will be denoted by $\hat{\mu}=$ $(\hat{a}, \hat{3}, \hat{m})$. The $S^{3}$ part is described by Hopf coordinates $x^{m}=\left(\theta_{1}, \theta_{2}, \theta_{3}\right)$. In the limit $\tau \rightarrow 0$ and $\kappa \rightarrow 0$, the $\operatorname{AdS}_{3}$ and $S^{3}$ become flat Minkowski space and flat space $\mathbb{R}^{3}$, respectively.
With the vielbeins on $\mathrm{AdS}_{3}$ and $S^{3}$ of the form

$$
\begin{aligned}
e^{\hat{0}} & =\frac{1}{\tau}\left(d t-\sinh x^{1} d x^{2}\right), \\
e^{\hat{1}} & =\frac{1}{\tau}\left(\cos t d x^{1}-\sin t \cosh x^{1} d x^{2}\right), \\
e^{\hat{2}} & =\frac{1}{\tau}\left(\sin t d x^{1}+\cos t \cosh x^{1} d x^{2}\right)
\end{aligned}
$$

and

$$
\begin{aligned}
e^{\hat{4}} & =\frac{1}{\kappa}\left(d \theta_{1}+\sin \theta_{2} d \theta_{3}\right), \\
e^{\hat{5}} & =\frac{1}{\kappa}\left(\cos \theta_{1} d \theta_{2}-\sin \theta_{1} \cos \theta_{2} d \theta_{3}\right), \\
e^{\hat{6}} & =\frac{1}{\kappa}\left(\sin \theta_{1} d \theta_{2}+\cos \theta_{1} \cos \theta_{2} d \theta_{3}\right),
\end{aligned}
$$

the spin connections take a simple form

$$
\begin{array}{rlrl}
\omega_{\hat{a}}^{\hat{a}} \hat{3} & =e^{-V} U^{\prime}, & \omega_{\hat{a} \hat{b} \hat{c}} & =\frac{\tau}{2} e^{-U} \epsilon_{\hat{a} \hat{b} \hat{c}}, \\
\omega_{\hat{m}}^{\hat{m}} \hat{\hat{3}}=e^{-V} W^{\prime}, & \omega_{\hat{m} \hat{n} \hat{p}}=\frac{\kappa}{2} e^{-W} \epsilon_{\hat{m} \hat{n} \hat{p}}
\end{array}
$$

with $\epsilon_{\hat{0} \hat{1} \hat{2}}=\epsilon_{\hat{4} \hat{5} \hat{b}}=1$. We will use a prime to denote the $r$ derivative throughout the paper.

As in the usual domain wall solutions, the scalar fields $\sigma$ and $\phi$ are functions of only $r$, while the ansatz for the threeform field is taken to be

$$
C_{(3)}=k(r) \mathrm{Vol}_{\mathrm{AdS}_{3}}+l(r) \mathrm{Vol}_{S^{3}}
$$

in which $\mathrm{Vol}_{\mathrm{AdS}_{3}}$ and $\mathrm{Vol}_{S^{3}}$ are volume forms on $\mathrm{AdS}_{3}$ and $S^{3}$, respectively. We will also set $A_{(1)}^{I}=0$, since, in this section, we are interested only in solutions with vanishing vector fields.

The ansatz for Killing spinors corresponding to the unbroken supersymmetry takes the form of

$$
\epsilon=Y(r)\left[\cos \theta(r) \mathbf{1}_{8}+\sin \theta(r) \gamma^{\hat{0} \hat{1} \hat{2}}\right] \epsilon_{0}
$$

with the constant spinor $\epsilon_{0}$ satisfying the projection condition

$$
\gamma^{\hat{3}} \epsilon_{0}=\epsilon_{0}
$$

$Y(r)$ and $\theta(r)$ are functions of $r$ to be determined.

To find supersymmetric solutions, we consider Bogomol'nyi-Prasad-Sommerfield (BPS) equations obtained from supersymmetry transformations of fermionic fields $\left(\psi_{\mu}, \chi, \lambda^{r}\right)$. Using the Killing spinor (38) and the projection (39), we obtain two equations from $\delta \lambda^{r}=0$ conditions: 


$$
\begin{aligned}
& P_{\hat{3}}^{i r} \cos 2 \theta-\frac{1}{\sqrt{2}} e^{-\frac{\sigma}{2}} C^{i r}=0, \\
& P_{\hat{3}}^{i r}-\frac{1}{\sqrt{2}} e^{-\frac{\sigma}{2}} C^{i r} \cos 2 \theta=0 .
\end{aligned}
$$

For the coset representative (23), we can readily compute $P_{\mu}^{i r}$ and $C^{i r}$. The result is given by

$$
\begin{aligned}
P_{\hat{3}}^{i r} & =\phi^{\prime} e^{-V} \delta^{i r} \quad \text { and } \\
C^{i r} & =\sqrt{2}\left(g_{1} \cosh \phi-g_{2} \sinh \phi\right) \cosh \phi \sinh \phi \delta^{i r} .
\end{aligned}
$$

Note also that the three-form field does not enter the $\delta \lambda^{r}$ equations.

Compatibility between Eqs. (40) and (41) implies $\cos (2 \theta)= \pm 1$ leading to $\sin \theta=0$ or $\cos \theta=0$. Up to a redefinition of $\epsilon_{0}$ to $\tilde{\epsilon}_{0}=\gamma^{\hat{0} \hat{1} \hat{2}} \epsilon_{0}$ and a sign change in the projection condition (39), the two choices give equivalent BPS equations. For definiteness, we will choose $\sin \theta=0$ in the following analysis. This leads to the BPS equation for $\phi$ :

$$
\phi^{\prime}=e^{V-\frac{\sigma}{2}}\left(g_{1} \cosh \phi-g_{2} \sinh \phi\right) \cosh \phi \sinh \phi .
$$

The Killing spinor then takes a simpler form

$$
\epsilon=Y(r) \epsilon_{0} .
$$

We now consider the $\delta \chi=0$ equation. This condition involves a contribution from the three-form field of the form $H_{\mu \nu \rho \sigma} \gamma^{\mu \nu \rho \sigma} \epsilon$. We will use the same convention for spinors and gamma matrices as in Ref. [23]. Using the relation $\gamma^{\hat{0}} \gamma^{\hat{1}} \gamma^{\hat{2}} \gamma^{\hat{3}} \gamma^{\hat{4}} \gamma^{\hat{5}} \gamma^{\hat{6}}=\mathbf{1}_{8}$ or more compactly $\epsilon_{\hat{a} \hat{b} \hat{c}} \gamma^{\hat{a} \hat{b} \hat{c}} \gamma^{\hat{r}}=-\epsilon_{\hat{m} \hat{n} \hat{p}} \gamma^{\hat{m} \hat{n} \hat{p}}$, we find

$$
\frac{1}{4 !} H_{\mu \nu \rho \sigma} \gamma^{\mu \nu \rho \sigma} \epsilon=\left(l^{\prime} e^{-V-3 W}-k^{\prime} e^{-V-3 U}\right) \gamma^{\hat{0} \hat{1} \hat{2}} \epsilon .
$$

Since there is no other term contributing a $\gamma^{\hat{0} \hat{1}} \hat{2}$ matrix in the $\delta \chi$ variation, this term must vanish by itself. This can be achieved by setting

$$
k^{\prime} e^{-3 U}=l^{\prime} e^{-3 W},
$$

which leads to the BPS equation for $\sigma$ :

$$
\sigma^{\prime}=-\frac{2}{5} e^{V-\frac{\sigma}{2}}\left[16 h e^{\frac{5}{2} \sigma}+g_{1} \cosh ^{3} \phi-g_{2} \sinh ^{3} \phi\right] .
$$

We then move on to the BPS equations from $\delta \psi_{\mu}$ conditions. After using the $\gamma^{\hat{r}}$ projection (39) and the three-form ansatz (37) in the conditions $\delta \psi_{a}=0$ and $\delta \psi_{m}=0$, we find two types of terms one with $\gamma^{\hat{0}} \hat{1} \hat{2}$ and the other with $\mathbf{1}_{8}$. The former gives rise to the BPS equations for $k$ and $l$ :

$$
k^{\prime}=\frac{\tau}{\sqrt{2}} e^{2 U+\sigma+V}, \quad l^{\prime}=\frac{\kappa}{\sqrt{2}} e^{2 W+\sigma+V},
$$

while the latter gives the corresponding equations for $U$ and $W$ :

$U^{\prime}=W^{\prime}=\frac{1}{5} e^{V-\frac{\sigma}{2}}\left[4 h e^{\frac{5}{2} \sigma}-g_{1} \cosh ^{3} \phi+g_{2} \sinh ^{3} \phi\right]$.

The last equation implies that $U=W+C$ for a constant $C$. In order to find solutions interpolating between $\mathrm{AdS}_{7}$ vacua, we require that the solutions be asymptotically locally $\mathrm{AdS}_{7}$ at which $U=W$. This implies that $C=0$ or $U=W$.

Using this relation in Eq. (46), we find that $k^{\prime}=l^{\prime}$ or $k=l+\tilde{C}$ for some constant $\tilde{C}$. This constant can be set to zero by a suitable redefinition of $k$ and $l$. We will accordingly set $k=l$. With all these, Eq. (48) gives

$$
\tau=\kappa
$$

In summary, we end up with the BPS equations for the warped factor $U$ and $k$ in the form of

$$
\begin{aligned}
U^{\prime} & =\frac{1}{5} e^{V-\frac{\sigma}{2}}\left[4 h e^{\frac{5}{2} \sigma}-g_{1} \cosh ^{3} \phi+g_{2} \sinh ^{3} \phi\right], \\
k^{\prime} & =\frac{\kappa}{\sqrt{2}} e^{2 U+\sigma+V} .
\end{aligned}
$$

It should be noted that the contribution from $C_{(3)}$ is canceled by the spin connections on $\operatorname{AdS}_{3}$ and $S^{3}$. Therefore, for nonvanishing $C_{(3)}$ and $k=l$, there can be no background with Mink $_{3}$ and $\mathbb{R}^{3}$. This is perfectly in agreement with a similar solution considered in Ref. [23] but without the scalar from vector multiplets. It can also be easily checked that any solutions to the above BPS equations solve all the field equations.

We finally consider the equation from the $\delta \psi_{3}$ condition. This gives the BPS equation for $Y(r)$ :

$$
Y^{\prime}=\frac{1}{2} Y U^{\prime}
$$

which can be solved by a solution $Y \sim e^{\frac{U}{2}}$.

We are now in a position to solve all of the BPS equations. To find an analytic solution, we first choose a function $V(r)=\frac{\sigma}{2}$. This is equivalent to changing to a new radial coordinate $\tilde{r}$ defined by the relation $\frac{d \tilde{r}}{d r}=e^{-\frac{\sigma}{2}}$ in Ref. [19]. The procedure is very similar to that used in Ref. [19], so we will not repeat all the details here. After choosing $V(r)=\frac{\sigma}{2}$, we obtain the solution for (43): 


$$
\begin{aligned}
g_{1} g_{2} r= & g_{2} \ln \left(1-e^{2 \phi}\right)-g_{1} \ln \left(1+e^{2 \phi}\right) \\
& +\frac{\left(g_{1}+g_{2}\right)^{2}}{g_{1}-g_{2}} \ln \left[g_{1}+g_{2}+\left(g_{1}-g_{2}\right) e^{2 \phi}\right],
\end{aligned}
$$

where an irrelevant additive integration constant has been neglected.

By treating $U, \sigma$, and $k$ as functions of $\phi$, we find the solution of Eqs. (47), (51), and (52):

$$
\begin{aligned}
\sigma= & \frac{2}{5} \ln \left[\frac{g_{1} g_{2}}{16 h\left(g_{1} \sinh \phi-g_{2} \cosh \phi\right)}\right], \\
U= & \frac{1}{4} \phi-\frac{1}{8} \sigma-\frac{1}{4} \ln \left(e^{4 \phi}-1\right) \\
& +\frac{1}{4} \ln \left[g_{1}+g_{2}+\left(g_{1}-g_{2}\right) e^{2 \phi}\right], \\
k= & \frac{\tau}{4}\left[\frac{g_{1}}{g_{2}}+\frac{g_{2}}{g_{1}}-2 \operatorname{coth}(2 \phi)\right],
\end{aligned}
$$

in which irrelevant integration constants in $U$ and $k$ have been removed. The integration constant in $\sigma$ is, however, important and has been chosen such that the solution for $\sigma$ interpolates between the two supersymmetric $\mathrm{AdS}_{7}$ critical points; see [19] for more detail.

As $r \rightarrow \pm \infty$, the solution is asymptotic to the $\mathrm{AdS}_{7}$ critical points with

$U \sim 4 h r, \quad \sigma \sim \phi \sim 0, \quad F_{\hat{0} \hat{1} \hat{2} \hat{r}} \sim F_{\hat{r} \hat{4} \hat{5} \hat{b}} \sim 0$

for $r \rightarrow \infty$ and

$$
\begin{aligned}
U & \sim 4 h\left(\frac{g_{2}^{2}}{g_{2}^{2}-256 h^{2}}\right)^{\frac{2}{5}} r, \quad \sigma \sim \frac{1}{5} \ln \left[\frac{g_{2}^{2}}{g_{2}^{2}+256 h^{2}}\right], \\
\phi & \sim \frac{1}{2} \ln \left[\frac{g_{2}-16 h}{g_{2}+16 h}\right], \quad F_{\hat{0} \hat{1} \hat{2} \hat{r}} \sim F_{\hat{r} \hat{4} \hat{5} \hat{b}} \sim 0
\end{aligned}
$$

for $r \rightarrow-\infty$. In these equations, we have set $g_{1}=-16 h$.

It should be noted that the four-form field strength does not actually vanish in the limit $r \rightarrow \pm \infty$ as can be seen from the BPS equation for $k^{\prime}$. Moreover, the existence of $C_{(3)}$ is needed to support the $\mathrm{AdS}_{3}$ and $S^{3}$ factors as mentioned above. However, its effect in the limit $r \rightarrow \pm \infty$ is highly suppressed compared to the scalar potential. The solution is then asymptotically locally $\mathrm{AdS}_{7}$ as $r \rightarrow \pm \infty$.

\section{B. Solutions with known higher-dimensional origin}

For a particular case of $g_{2}=g_{1}$, solutions of the $N=2$ gauged supergravity can be uplifted to 11 dimensions. The corresponding reduction ansatz has been constructed in Ref. [27]. Setting $g_{2}=g_{1}$, we obtain the BPS equations

$$
\begin{aligned}
\phi^{\prime} & =e^{V-\frac{\sigma}{2}-\phi} g_{1} \cosh \phi \sinh \phi, \\
\sigma^{\prime} & =-\frac{2}{5} e^{V-\frac{\sigma}{2}}\left[16 h e^{\frac{5}{2} \sigma}+g_{1} \cosh ^{3} \phi-g_{1} \sinh ^{3} \phi\right], \\
U^{\prime} & =\frac{1}{5} e^{V-\frac{\sigma}{2}}\left[4 h e^{\frac{5}{2} \sigma}-g_{1} \cosh ^{3} \phi+g_{1} \sinh ^{3} \phi\right], \\
k^{\prime} & =\frac{\kappa}{\sqrt{2}} e^{2 U+\sigma+V} .
\end{aligned}
$$

It can be clearly seen from the $\phi^{\prime}$ equation that there is only one supersymmetric $\mathrm{AdS}_{7}$ background at $\phi=0$. The solutions interpolating between this $\mathrm{AdS}_{7}$ and physically acceptable, singular geometries dual to nonconformal field theories in the case of $k=0$ have already been studied in Ref. [27]. In this paper, we will give the solution with a nonvanishing three-form field. This solution can be found by the analysis similar to the previous case. The resulting solution is given by

$$
\begin{aligned}
g_{1} r & =2 \tan ^{-1} e^{\phi}-2 \tanh ^{-1} e^{\phi}, \\
\sigma & =\frac{2}{5} \phi-\frac{2}{5} \ln \left[1-12 C_{1}\left(e^{4 \phi}-1\right)\right], \\
U & =\frac{1}{5} \phi-\frac{1}{4} \ln \left(e^{4 \phi}-1\right)+\frac{1}{20} \ln \left[1-12 C_{1}\left(e^{4 \phi}-1\right)\right], \\
k & =\frac{\tau}{2 h}\left(\frac{h^{4}}{2^{9} g_{1}^{4}}\right)^{\frac{1}{10}} \sqrt{\frac{1-12 C_{1}\left(e^{4 \phi}-1\right)}{e^{4 \phi}-1}} .
\end{aligned}
$$

It can be seen that $\phi$ diverges at a finite value of $r$. Therefore, the solution is singular at this point. Without the loss of generality, we can shift the coordinate $r$ such that the singularity occurs at $r=0$. The integration constant $C_{1}$ controls the behavior near the singularity; see [27] for more detail.

For $C_{1}=0$, the solution near $r=0$ becomes

$$
\begin{aligned}
\phi & \sim-\ln (4 h r), \quad \sigma \sim-\frac{2}{5} \ln (4 h r), \quad k \sim e^{-2 \phi} \sim(4 h r)^{2}, \\
d s_{7}^{2} & =(4 h r)^{2}\left(d s_{\mathrm{AdS}_{3}}^{2}+d s_{S^{3}}^{2}\right)+(4 h r)^{-\frac{1}{5}} d r^{2},
\end{aligned}
$$

in which we have set $g_{1}=-16 h$. For $C_{1} \neq 0$, we find

$$
\begin{aligned}
\phi & \sim-\ln (4 h r), \quad \sigma \sim \frac{6}{5} \ln (4 h r), \quad k \sim \text { constant }, \\
d s_{7}^{2} & =(4 h r)^{\frac{3}{4}}\left(d s_{\mathrm{AdS}_{3}}^{2}+d s_{S^{3}}^{2}\right)+(4 h r)^{\frac{3}{5}} d r^{2} .
\end{aligned}
$$

As pointed out in Ref. [27], all of these singularities are physically acceptable, since the scalar potential is bounded from above, in this case $V \rightarrow-\infty$, as required by the criterion in Ref. [28]. 
In this case, the solution can be embedded in 11 dimensions by using the reduction ansatz in Ref. [27]. For convenience, we give a brief review of this result in Appendix B. The nine scalars from vector multiplets can be equivalently described by $S L(4, \mathbb{R}) / S O(4)$ coset due to the isomorphism $S L(4, \mathbb{R}) \sim S O(3,3)$. For the $S O(3)_{\mathrm{diag}}$ singlet scalar, we find the $S L(4, \mathbb{R}) / S O(4)$ coset representative

$$
V_{\alpha}^{R}=\operatorname{diag}\left(e^{\frac{\phi}{2}}, e^{\frac{\phi}{2}}, e^{\frac{\phi}{2}}, e^{-\frac{3 \phi}{2}}\right)
$$

which gives a symmetric $4 \times 4$ matrix with unit determinant

$$
\tilde{T}_{\alpha \beta}=\operatorname{diag}\left(e^{\phi}, e^{\phi}, e^{\phi}, e^{-3 \phi}\right)=\left(\delta_{a b} e^{\phi}, e^{-3 \phi}\right) .
$$

In the remaining parts of this section, we will use indices $a, b=1,2,3$ to denote coordinates $\hat{\mu}^{a}$ on the internal $S^{2}$ with $\hat{\mu}^{a} \hat{\mu}^{a}=1$. We will also use the $S^{3}$ coordinates $\mu^{\alpha}=$ $\left(\cos \psi \hat{\mu}^{a}, \sin \psi\right)$ satisfying $\mu^{\alpha} \mu^{\alpha}=1$.

With all these and the seven-dimensional fields given previously, we obtain the 11-dimensional metric

$$
\begin{aligned}
d \hat{s}_{11}^{2}= & \Delta^{\frac{1}{3}}\left[e^{2 U}\left(d s_{\mathrm{AdS}_{3}}^{2}+d s_{S^{3}}^{2}\right)+e^{2 V} d r^{2}\right]+\frac{1}{32 h^{2}} \Delta^{-\frac{2}{3}} e^{-2 \sigma}\left[\cos ^{2} \xi+e^{\frac{5}{2} \sigma} \sin ^{2} \xi\left(e^{-\phi} \cos ^{2} \psi+e^{3 \phi} \sin ^{2} \psi\right)\right] d \xi^{2} \\
& +\frac{1}{64 h^{2}} \Delta^{-\frac{2}{3}} e^{\frac{\sigma}{2}} \sin \xi \sin \psi \cos \psi\left(e^{-\phi}-e^{3 \phi}\right) d \xi d \psi \\
& +\frac{1}{128 h^{2}} \Delta^{-\frac{2}{3}} e^{\frac{\sigma}{2}} \cos ^{2} \xi\left[\left(e^{3 \phi} \cos ^{2} \psi+e^{-\phi} \sin ^{2} \xi\right) d \psi^{2}+e^{-\phi} \cos ^{2} \psi d \Omega_{2}^{2}\right]
\end{aligned}
$$

with the warped factor given by

$$
\Delta=e^{-\frac{\sigma}{2}} \cos ^{2} \xi\left(e^{\phi} \cos ^{2} \psi+e^{-3 \phi} \sin ^{2} \psi\right)+e^{2 \sigma} \sin ^{2} \xi,
$$

and the metric on a unit two-sphere can be written as $d \Omega_{2}^{2}=d \hat{\mu}^{a} d \hat{\mu}^{a}$. It should be noted that the $S^{2}$ in the internal $S^{3}$ is unchanged. Its isometry corresponds to the unbroken $S O(3)_{\text {diag }}$ symmetry.

The four-form field strength of 11-dimensional supergravity is given by

$$
\begin{aligned}
\hat{F}_{(4)}= & \sin \xi \sqrt{2} k^{\prime}\left(d r \wedge \mathrm{Vol}_{\mathrm{AdS}_{3}}+d r \wedge \mathrm{Vol}_{S^{3}}\right)-\frac{\sqrt{2}}{8 h} e^{-2 \sigma} \cos \xi k^{\prime} e^{-V}\left(\mathrm{Vol}_{\mathrm{AdS}_{3}}+\mathrm{Vol}_{S^{3}}\right) \wedge d \xi \\
& +\frac{1}{(8 h)^{3}} \Delta^{-2} U \cos ^{3} \xi \cos ^{2} \psi d \xi \wedge d \psi \wedge \epsilon_{(2)} \\
& +\frac{1}{(8 h)^{3}} \Delta^{-2} e^{\frac{3}{2} \sigma} \sin \xi \cos ^{4} \xi \cos ^{2} \psi\left[e^{\phi} \cos ^{2} \psi\left(\phi^{\prime}-\frac{5}{2} \sigma^{\prime}\right)-e^{-3 \phi} \sin ^{2} \psi\left(\frac{5}{2} \sigma^{\prime}+3 \phi^{\prime}\right)\right] d r \wedge d \psi \wedge \epsilon_{(2)} \\
& -\frac{1}{(8 h)^{3}} \Delta^{-2} \cos ^{2} \xi \cos ^{3} \psi \sin \psi\left[\left[4 e^{-2 \phi-\sigma} \cos ^{3} \xi+e^{\frac{3}{2} \sigma}\left(e^{\phi}+3 e^{-3 \phi}\right)\right] \phi^{\prime}-\frac{5}{2} \sin ^{2} \xi e^{\frac{3}{2} \sigma}\left(e^{\phi}-e^{-3 \phi}\right) \sigma^{\prime}\right] d r \wedge d \xi \wedge \epsilon_{(2)},
\end{aligned}
$$

where $\epsilon_{(2)}=\frac{1}{2} \epsilon_{a b c} \hat{\mu}^{a} d \hat{\mu}^{b} d \hat{\mu}^{c}$ is the volume form on $S^{2}$. In this equation, we have also used $\epsilon_{a b c 4}=\epsilon_{a b c}$. The scalar function $U$ is given by

$$
\begin{aligned}
U= & \sin ^{2} \xi\left(e^{4 \sigma}-3 e^{\phi+\frac{3}{2} \sigma}-e^{\frac{3}{2} \sigma-3 \phi}\right)-\cos ^{2} \xi\left[e^{\frac{3}{2} \sigma}\left(e^{\phi} \cos ^{2} \psi+e^{-3 \phi} \sin ^{2} \psi\right)\right. \\
& \left.+e^{-\sigma}\left(e^{2 \phi} \cos ^{2} \psi+3 e^{2 \phi} \sin ^{2} \psi+e^{-2 \phi} \cos ^{2} \psi-e^{-6 \phi} \sin ^{2} \psi\right)\right] .
\end{aligned}
$$

Similar to the discussion in Ref. [23], we expect the uplifted solution to describe 11-dimensional configurations involving M2-M5-brane bound states due to the dyonic profile of $C_{(3)}$. It is also interesting to consider the (00) component of the 11-dimensional metric:

$$
\hat{g}_{00}=-\frac{1}{\kappa^{2}} \Delta^{\frac{1}{3}} e^{2 U(r)} .
$$

Near the singularity at $r=0$, we find that

$$
\hat{g}_{00} \sim(4 h r)^{\frac{26}{15}} \rightarrow 0 \quad \text { and } \quad \hat{g}_{00} \sim(4 h r)^{\frac{13}{60}} \rightarrow 0
$$

for $C_{1}=0$ and $C_{1} \neq 0$, respectively. According to the criterion of Ref. [29], the singularities are physical in agreement with the seven-dimensional results obtained from the criterion of Ref. [28]. We then expect that the 
solution holographically describes a two-dimensional conformal defect in six-dimensional $N=(1,0)$ SCFT with known M-theory origin.

\section{DOMAIN WALLS WITH THE THREE-FORM AND VECTOR FIELDS}

In this section, we consider more general solutions with nonvanishing vector fields. We first choose an appropriate ansatz for the $S O(4) \sim S O(3) \times S O(3)$ gauge fields. As in Ref. [23], we will take this ansatz in the form of

$$
A_{(1)}^{I}=A_{i}^{I} d \theta_{i}
$$

in which the components $A_{i}^{I}$ will be functions of only the radial coordinate $r$. Explicitly, these components are given by

$$
\begin{aligned}
& A_{j}^{i}=-\frac{e^{-W} \kappa}{2} A(r) \delta_{j}^{i}, \\
& A_{i}^{r}=-\frac{e^{-W} \kappa}{2} B(r) \delta_{i}^{r} .
\end{aligned}
$$

It is now straightforward to compute the field strength tensors $F_{(2)}^{i}=L_{I}{ }^{i} F_{(2)}^{I}$ and $F_{(2)}^{r}=L_{I}{ }^{r} F_{(2)}^{I}$. Nonvanishing components of these tensors are given by

$$
\begin{aligned}
F_{3 j}^{i}=\mathbf{f} \delta_{j}^{i}, & F_{j k}^{i}=\mathbf{g} \epsilon_{i j k}, \\
F_{3 i}^{r}=\overline{\mathbf{f}} \delta_{i}^{r}, & F_{j k}^{r}=\overline{\mathbf{g}} \delta_{i}^{r} \epsilon_{i j k},
\end{aligned}
$$

where

$\mathbf{f}=-e^{-V-W} \frac{\kappa}{2}\left[A^{\prime} \cosh \phi+B^{\prime} \sinh \phi\right]$,

$\overline{\mathbf{f}}=-e^{-V-W} \frac{\kappa}{2}\left[A^{\prime} \sinh \phi+B^{\prime} \cosh \phi\right]$,

$\mathbf{g}=-e^{-2 W} \frac{\kappa^{2}}{4}\left[A\left(2-g_{1} A\right) \cosh \phi+B\left(2+g_{2} B\right) \sinh \phi\right]$,

$\overline{\mathbf{g}}=-e^{-2 W} \frac{\kappa^{2}}{4}\left[A\left(2-g_{1} A\right) \sinh \phi+B\left(2+g_{2} B\right) \cosh \phi\right]$.

To implement $S O(3)_{\text {diag }}$, we will set

$$
g_{2} B=-g_{1} A .
$$

We still use the ansatz for the Killing spinor as given in (38) and the projection (39). Because of the extra contributions from nonvanishing gauge fields, we need more projectors

$$
\gamma^{\hat{5} \hat{b}} \epsilon=-i \sigma^{1} \epsilon, \quad \gamma^{\hat{4} \hat{b}} \epsilon=i \sigma^{2} \epsilon, \quad \gamma^{\hat{4} \hat{5}} \epsilon=-i \sigma^{3} \epsilon .
$$

The second condition is just the symplectic-Majorana condition. Therefore, the BPS solutions (if any exist) will preserve only two supercharges or $\frac{1}{8}$ BPS after imposing the projection (39).

With all these, we can now set up the BPS equations. By the relation (87), the composite connection along $S^{3}$ takes a very simple form

$$
Q_{i j k}=\omega_{i+3, j+3, k+3},
$$

where $\omega_{i+3, j+3, k+3}$ is the spin connection given in (36). Using the same procedure as in the previous section, we find the following set of BPS equations:

$$
\begin{aligned}
U^{\prime}= & \frac{e^{V}}{60 \cos 2 \theta}\left[2\left[12 h e^{2 \sigma}+\frac{e^{-\frac{\sigma}{2}}}{\sqrt{2}} C\right](3 \cos 4 \theta-1)\right. \\
& +18 e^{\frac{\sigma}{2}} \mathbf{g}(\cos 4 \theta-3)+24 e^{-U} \tau \sin 2 \theta \\
& \left.+18 e^{-W} \kappa\left(g_{1} A-1\right) \sin 4 \theta\right],
\end{aligned}
$$

$$
\begin{aligned}
W^{\prime}= & -\frac{e^{V}}{30 \cos 2 \theta}\left[2\left[12 h e^{2 \sigma}+\frac{e^{-\frac{\sigma}{2}}}{\sqrt{2}} C\right](\cos 4 \theta-2)\right. \\
& +6 e^{\frac{\sigma}{2}} \mathbf{g}(\cos 4 \theta-8)+18 e^{-U} \tau \sin 2 \theta \\
& \left.+6 e^{-W} \kappa\left(g_{1} A-1\right) \sin 4 \theta\right]
\end{aligned}
$$

$$
\begin{aligned}
Y^{\prime}= & \frac{e^{V} Y}{120 \cos 2 \theta}\left[2\left[12 h e^{2 \sigma}+\frac{e^{-\frac{\sigma}{2}}}{\sqrt{2}} C\right](3 \cos 4 \theta-1)\right. \\
& +18 e^{\frac{\sigma}{2}} \mathbf{g}(\cos 4 \theta-3)+24 e^{-U} \tau \sin 2 \theta \\
& \left.+18 e^{-W} \kappa\left(g_{1} A-1\right) \sin 4 \theta\right], \\
\theta^{\prime}= & \frac{e^{V}}{4}\left[-2\left[4 h e^{2 \sigma}+\frac{e^{-\frac{\sigma}{2}}}{\sqrt{2}} C\right] \sin 2 \theta-6 e^{\frac{\sigma}{2}} \mathbf{g} \sin 2 \theta+6 e^{-U} \tau\right. \\
& \left.+6 e^{-W} \kappa\left(g_{1} A-1\right) \cos 2 \theta\right],
\end{aligned}
$$

$k^{\prime}=\frac{e^{3 U+V} e^{\sigma}}{3 \sqrt{2}}\left[2\left[12 h e^{2 \sigma}+\frac{e^{-\frac{\sigma}{2}}}{\sqrt{2}} C\right] \tan 2 \theta+18 e^{\frac{\sigma}{2}} \mathbf{g} \tan 2 \theta\right.$

$$
\left.-6 e^{-U} \tau \sec 2 \theta-9 e^{-W} \kappa\left(g_{1} A-1\right)\right],
$$

$l^{\prime}=\frac{1}{\sqrt{2}} e^{3 W+V} e^{\sigma}\left[e^{-U} \tau-8 h e^{2 \sigma} \sin 2 \theta\right]$, 


$$
\begin{aligned}
\sigma^{\prime}= & -\frac{e^{V}}{30 \cos 2 \theta}\left[48 h e^{2 \sigma}(\cos 4 \theta+3)\right. \\
& -\sqrt{2} C e^{-\frac{\sigma}{2}}(3 \cos 4 \theta-1)-24 e^{-U} \tau \sin 2 \theta \\
& \left.-18 e^{\frac{\sigma}{2}} \mathbf{g}(\cos 4 \theta-3)-18 e^{-W} \kappa\left(g_{1} A-1\right) \sin 4 \theta\right] \\
\phi^{\prime} & =e^{V}\left[\frac{e^{-\frac{\sigma}{2}}}{\sqrt{2}} \mathcal{C}-e^{\frac{\sigma}{2} \mathbf{g}}\right] \cos 2 \theta \\
A^{\prime}= & -\frac{2 g_{2} e^{V+W-\frac{\sigma}{2}}}{\kappa\left(g_{1} \sinh \phi-g_{2} \cosh \phi\right)}\left[\frac{1}{3 \sqrt{2}} C e^{-\frac{\sigma}{2}} \sin 2 \theta+e^{\frac{\sigma}{2}} \mathbf{g} \sin 2 \theta\right. \\
& \left.-\left[e^{-U} \tau+e^{-W} \kappa\left(g_{1} A-1\right) \cos 2 \theta\right]\right]
\end{aligned}
$$

where the quantities $C$ and $\mathcal{C}$ are defined, respectively, by

$$
\begin{aligned}
& C=-3 \sqrt{2}\left(g_{1} \cosh ^{3} \phi-g_{2} \sinh ^{3} \phi\right), \\
& \mathcal{C}=\frac{1}{\sqrt{2}} \sinh (2 \phi)\left(g_{1} \cosh \phi-g_{2} \sinh \phi\right) .
\end{aligned}
$$

In addition to these flow equations, there is an algebraic constraint arising from the fact that the supersymmetry transformations from the gravity multiplet $\left(\delta \psi_{\mu}\right.$ and $\left.\delta \chi\right)$ and those from the vector multiplets $\left(\delta \lambda^{r}\right)$ lead to different BPS equations for $A$. Consistency between these two equations results in a constraint

$$
\begin{aligned}
0= & e^{\frac{\sigma}{2}} \sin 2 \theta\left[\left(\frac{e^{-\sigma}}{3 \sqrt{2}} C+\mathbf{g}\right)\right. \\
& \left.+\frac{g_{1} \sinh \phi-g_{2} \cosh \phi}{g_{1} \cosh \phi-g_{2} \sinh \phi}\left(-\frac{e^{-\sigma}}{\sqrt{2}} \mathcal{C}+\overline{\mathbf{g}}\right)\right] \\
& +e^{-W} \kappa\left(1-g_{1} A\right) \cos 2 \theta-e^{-U} \tau .
\end{aligned}
$$

This means supersymmetric solutions must satisfy the above BPS equations as well as the constraint (101) in order for the Killing spinors to exist. We have explicitly verified that the BPS equations (90)-(98) together with the constraint (101) imply all of the field equations.

However, it turns out that there are no solutions with nontrivial three-form and vector fields satisfying the BPS equations (90)-(98) and the constraint (101). This can be readily checked by differentiating Eq. (101) and substituting the BPS equations (90)-(98). The result gives the following condition:

$0=e^{-2 U-\frac{\sigma}{2}} k^{\prime} \mathbf{g}-\frac{2 e^{U+\frac{\sigma}{2}}\left(g_{2}^{2}-g_{1}^{2}\right) \phi^{\prime} \mathbf{f}}{\left(g_{2} \sinh \phi-g_{1} \cosh \phi\right)\left(g_{2} \cosh \phi-g_{1} \sinh \phi\right)}$,

which cannot be satisfied by the BPS equations (94) and (97). This implies that $\phi$ and $k$ cannot flow independently but relate to each other by Eq. (102).
Note that relation (102) is trivially satisfied for $A=0$, which gives $\mathbf{f}=\mathbf{g}=0$. This results in the BPS equations considered in the previous section. Another possibility is to set $k^{\prime}=0$ and $g_{2}^{2}=g_{1}^{2}$. However, with $k^{\prime}=0$ and the constraint (101), Eq. (94) implies $\theta=0$ and $A=\frac{1}{g_{1}}$ for generic values of $\sigma$ and $\phi$. Although this result is compatible with the BPS equations given in (93) and (98), it also leads to $l^{\prime}=0$ as can be seen from Eq. (95) after using the constraint (101). The three-form field then has vanishing field strength. In this case, the gauge fields do not depend on the radial coordinate $r$. This type of solution has already been studied in Ref. [21] in the context of twisted compactifications. Therefore, we conclude that there are no supersymmetric solutions with nonvanishing $S O(3)_{\text {diag }}$ gauge fields and a nontrivial three-form field.

\section{CONCLUSIONS AND DISCUSSIONS}

We have studied supersymmetric solutions of mattercoupled $N=2$ gauged supergravity in seven dimensions with the $S O(4) \sim S O(3) \times S O(3)$ gauge group. The resulting solutions are generalizations of the previously known solutions of $N=2$ gauged supergravity in the sense that all possible bosonic fields, from both gravity and vector multiplets, are considered. These solutions take the form of asymptotically locally $\mathrm{AdS}_{7}$ solutions and should be useful in the holographic study of $N=(1,0)$ six-dimensional SCFTs.

For vanishing vector fields, we have found analytic solutions to the BPS equations for all the fields that are singlets of the residual $S O(3)_{\text {diag }} \subset S O(3) \times S O(3)$ symmetry. For special values of $S O(3) \times S O(3)$ gauge couplings, namely, $g_{2}=g_{1}$, the solutions can be uplifted to 11 dimensions. We have performed this uplift and given the explicit form of the 11-dimensional metric and the fourform field strength tensor. Unlike the solutions found in Ref. [23], we have found that the solutions to the mattercoupled gauged supergravity are more restrictive. As a result, only solutions in the form of $\mathrm{AdS}_{3} \times S^{3}$ sliceddomain walls are possible. From a general notion of the AdS/CFT correspondence and the recent result in Ref. [25], we expect these solutions to describe some supersymmetric, two-dimensional, conformal defects in $N=(1,0)$ SCFT with flavor symmetry $S O(3)$. It is interesting to find the dual descriptions of these solutions in the $N=(1,0)$ SCFT. A generalization of these solutions to include more scalars, such as those invariant under smaller residual symmetries, is straightforward, since the three-form field does not couple directly to scalars from vector multiplets.

We have also derived a set of first-order flow equations together with an algebraic constraint for the case of nonvanishing $S O(3)_{\text {diag }}$ gauge fields. In this case, we have performed the analysis and argued that supersymmetric solutions do not exist at least within the truncation 
considered here. This is due to the fact that, in general, the constraint, arising from the supersymmetry variations of the gaugini, is violated by the solutions of the flow equations.

Solutions in $N=2$ gauged supergravity with other gauge groups and slicing different from $\mathrm{AdS}_{3} \times S^{3}$ are worth considering. Similar solutions in the maximal $N=4$ gauged supergravity in seven dimensions are also of particular interest. These would describe lower-dimensional defects within $N=(2,0)$ SCFT dual to the $\operatorname{AdS}_{7} \times S^{4}$ solution of $\mathrm{M}$ theory. We hope to come back to these issues in future works.

\section{ACKNOWLEDGMENTS}

We thank Giuseppe Dibitetto and Davide Cassani for helpful correspondences and clarifications. This work is supported by The Thailand Research Fund (TRF) under Grant No. RSA5980037.

\section{APPENDIX A: BOSONIC FIELD EQUATIONS OF $N=2$ GAUGED SUPERGRAVITY COUPLED TO VECTOR MULTIPLETS}

In this Appendix, we give all of the bosonic field equations obtained from the Lagrangian given in (8). These equations read

$$
\begin{gathered}
d\left(e^{-2 \sigma} * H_{(4)}\right)+8 h H_{(4)}-\frac{1}{\sqrt{2}} F_{(2)}^{I} \wedge F_{(2)}^{I}=0, \quad \text { (A1) } \\
\frac{5}{4} d * d \sigma-\frac{1}{2} e^{\sigma} a_{I J} * F_{(2)}^{I} \wedge F_{(2)}^{J}+e^{-2 \sigma} * H_{(4)} \wedge H_{(4)} \\
+\left[\frac{1}{4} e^{-\sigma}\left(C^{i r} C_{i r}-\frac{1}{9} C^{2}\right)+2 \sqrt{2} h e^{\frac{3}{2} \sigma} C-64 h^{2} e^{4 \sigma}\right] \epsilon_{(7)} \\
=0, \\
D\left(e^{\sigma} a_{I J} * F_{(2)}^{I}\right)-\sqrt{2} H_{(4)} \wedge F_{(2)}^{J}+* P^{i r} f_{I J}{ }^{K} L^{I}{ }_{r} L_{i K}=0,
\end{gathered}
$$

$$
\begin{array}{rl}
D & * P^{i r}-2 e^{\sigma} L_{I}^{i} L^{r}{ }_{J} * F_{(2)}^{I} \wedge F_{(2)}^{J} \\
& -\left[\frac{1}{\sqrt{2}} e^{-\sigma} C_{j r} C^{k r s} \epsilon^{i j k}+4 \sqrt{2} h e^{\frac{3 \sigma}{2}} C_{i r}\right] \epsilon_{(7)}=0, \\
R_{\mu \nu} & -\frac{5}{4} \partial_{\mu} \sigma \partial^{\mu} \sigma-P_{\mu}^{i r} P_{\nu i r}-\frac{2}{5} g_{\mu \nu} V \\
& -a_{I J} e^{\sigma}\left(F_{\mu \rho}^{I} F_{\nu}^{J \rho}-\frac{1}{10} g_{\mu \nu} F_{\rho \sigma}^{I} F^{J \rho \sigma}\right) \\
& -\frac{1}{6} e^{-2 \sigma}\left(H_{\mu \rho \sigma \lambda} H_{\nu}{ }^{\rho \sigma \lambda}-\frac{3}{20} g_{\mu \nu} H_{\rho \sigma \lambda \tau} H^{\rho \sigma \lambda \tau}\right)=0,
\end{array}
$$

where $C_{i r s}$ is defined by

$$
C_{i r s}=f_{I J}{ }^{K} L_{r}^{I} L_{s}^{J} L_{K i}
$$

The Yang-Mills equation (A3) can also be written in terms of $C^{i r}$ and $C^{i r s}$ by using the relation

$$
f_{I J}{ }^{K} L^{I}{ }_{r} L_{i K}=-\frac{1}{2 \sqrt{2}} \epsilon^{i j k} C^{j r} L^{k}{ }_{J}-C^{i r s} L_{S J}
$$

In deriving the scalar field equation (A4), it is useful to adopt the following projections, given in Ref. [13], in the form of

$$
\begin{aligned}
& \delta L^{i}{ }_{I}=X^{i}{ }_{r} L^{r}{ }_{I}+X^{i}{ }_{j} L^{j}{ }_{I}, \\
& \delta L^{r}{ }_{I}=X^{r}{ }_{s} L^{s}{ }_{I}+X^{r}{ }_{i} L^{i}{ }_{I} .
\end{aligned}
$$

With these relations, variations with respect to the scalar fields lead to the following results:

$$
\begin{aligned}
\delta C^{2} & =-6 C C^{i r} X_{i r}, \\
\delta\left(C^{i r} C_{i r}\right) & =2 \sqrt{2} C_{j s} C^{k r s} \epsilon^{i j k} X_{r}^{i}-\frac{2}{3} C_{i r} C X^{i}{ }_{r} .
\end{aligned}
$$

\section{APPENDIX B: REDUCTION ANSATZ FROM 11 DIMENSIONS}

In this Appendix, we summarize all relevant formulas for embedding seven-dimensional solutions in 11-dimensional supergravity. The reduction ansatz given in Ref. [27] is obtained from a truncation of the $S^{4}$ reduction giving rise to the maximal $N=4 S O(5)$ gauged supergravity [3]. The reduction gives an effective seven-dimensional $N=2$ gauged supergravity coupled to three vector multiplets and the $S O(4)$ gauge group. Because of the isomorphism $S O(3,3) \sim S L(4, \mathbb{R})$, the nine scalars from the vector multiplets can be equivalently parametrized by the $S L(4, \mathbb{R}) / S O(4)$ coset manifold. We follow all the conventions of Ref. [27], to which the reader is referred for more detail.

Let $V_{\alpha}{ }^{R}$ be $S L(4, \mathbb{R}) / S O(4)$ coset representative with $\alpha$, $R=1,2,3,4$. The bosonic part of the $N=2$ gauged supergravity is more conveniently described by a symmetric $4 \times 4$ matrix $\tilde{T}_{\alpha \beta}=V_{\alpha}{ }^{R} V_{\beta}{ }^{S} \delta_{R S}$ of unit determinant. The 11-dimensional metric and the four-form field of 11-dimensional supergravity are given, respectively, by

$$
\begin{aligned}
d \hat{s}_{11}^{2}= & \Delta^{\frac{1}{3}} d s_{7}^{2}+\frac{2}{g^{2}} \Delta^{-\frac{2}{3}} X^{3}\left[X \cos ^{2} \xi+X^{-4} \sin ^{2} \xi \tilde{T}_{\alpha \beta}^{-1} \mu^{\alpha} \mu^{\beta}\right] d \xi^{2} \\
& -\frac{1}{g^{2}} \Delta^{-\frac{2}{3}} X^{-1} \tilde{T}_{\alpha \beta}^{-1} \sin \xi \mu^{\alpha} d \xi D \mu^{\beta} \\
& +\frac{1}{2 g^{2}} \Delta^{-\frac{2}{3}} X^{-1} \tilde{T}_{\alpha \beta}^{-1} \cos ^{2} \xi D \mu^{\alpha} D \mu^{\beta}
\end{aligned}
$$

and 


$$
\begin{aligned}
\hat{F}_{(4)}= & F_{(4)} \sin \xi+\frac{1}{g} X^{4} \cos \xi * F_{(4)} \wedge d \xi+\frac{1}{g^{3}} \Delta^{-2} U \cos ^{5} \xi d \xi \wedge \epsilon_{(3)} \\
& +\frac{1}{3 ! g^{3}} \epsilon_{\alpha \beta \gamma \delta} \Delta^{-2} X^{-3} \sin \xi \cos ^{4} \xi \mu^{\kappa}\left[5 \tilde{T}^{\alpha \kappa} X^{-1} d X+D \tilde{T}^{\alpha \kappa}\right] \wedge D \mu^{\beta} \wedge D \mu^{\gamma} \wedge D \mu^{\delta} \\
& +\frac{1}{2 g^{2}} \cos \xi \epsilon_{\alpha \beta \gamma \delta}\left[\frac{1}{2} \cos \xi \sin \xi X^{-4} D \mu^{\gamma}-\left(X^{-4} \sin ^{2} \xi \mu^{\gamma}+X^{2} \cos ^{2} \xi \tilde{T}^{\gamma \kappa} \mu^{\kappa}\right) d \xi\right] \wedge F_{(2)}^{\alpha \beta} \wedge D \mu^{\delta} \\
& +\frac{1}{2 g^{3}} \epsilon_{\alpha \beta \gamma \delta} \Delta^{-2} \cos ^{3} \xi \mu^{\kappa} \mu^{\lambda}\left[\cos ^{2} \xi X^{2} \tilde{T}^{\alpha \kappa} D \tilde{T}^{\beta \lambda}-\sin ^{2} \xi X^{-3} \delta^{\beta \lambda} D \tilde{T}^{\alpha \kappa}-5 \sin ^{2} \xi \tilde{T}^{\alpha \kappa} X^{-4} \delta^{\beta \lambda} d X\right] \wedge D \mu^{\gamma} \wedge D \mu^{\delta} \wedge d \xi
\end{aligned}
$$

where $D \mu^{\alpha}=d \mu^{\alpha}+g A_{(1)}^{\alpha \beta} \mu^{\beta}$ and

$$
\begin{aligned}
U= & \sin ^{2} \xi\left(X^{-8}-X^{-3} \tilde{T}_{\alpha \alpha}\right) \\
& +\cos ^{2} \xi \mu^{\alpha} \mu^{\beta}\left(2 X^{2} \tilde{T}_{\alpha \gamma} \tilde{T}_{\gamma \beta}-X^{2} \tilde{T}_{\alpha \beta} \tilde{T}_{\gamma \gamma}-X^{-3} \tilde{T}_{\alpha \beta}\right), \\
\Delta= & \cos ^{2} \xi X \tilde{T}_{\alpha \beta} \mu^{\alpha} \mu^{\beta}+X^{-4} \sin ^{2} \xi, \\
\epsilon_{(3)}= & \frac{1}{3 !} \epsilon_{\alpha \beta \gamma \delta} \mu^{\alpha} D \mu^{\beta} \wedge D \mu^{\gamma} \wedge D \mu^{\delta} .
\end{aligned}
$$

The seven-dimensional fields and parameters are identified as follows:

$$
g_{2}=g_{1}=-16 h=-2 g, \quad X=e^{-\frac{\sigma}{2}}, \quad H_{(4)}=\frac{1}{\sqrt{2}} F_{(4)} .
$$

Relations involving the $S O(4)$ gauge fields are more complicated. Since, in this work, we do not consider the explicit embedding of solutions with nonvanishing gauge fields, we will not give them here.
[1] J. M. Maldacena, The large $N$ limit of superconformal field theories and supergravity, Adv. Theor. Math. Phys. 2, 231 (1998).

[2] K. Pilch, P. van Nieuwenhuizen, and P. K. Townsend, Compactification of $d=11$ supergravity on $S(4)$ (or $11=7+4$, too), Nucl. Phys. B242, 377 (1984).

[3] H. Nastase, D. Vaman, and P. van Nieuwenhuizen, Consistent nonlinear KK reduction of $11-d$ supergravity on $\operatorname{AdS}(7) \times S(4)$ and selfduality in odd dimensions, Phys. Lett. B 469, 96 (1999).

[4] M. Berkooz, A supergravity dual of a $(1,0)$ field theory in six dimensions, Phys. Lett. B 437, 315 (1998).

[5] S. Ferrara, A. Kehagias, H. Partouche, and A. Zaffaroni, Membranes and fivebranes with lower supersymmetry and their AdS supergravity duals, Phys. Lett. B 431, 42 (1998).

[6] C. Ahn, K. Oh, and R. Tatar, Orbifolds $\mathrm{AdS}_{7} \times S^{4}$ and sixdimensional (0,1) SCFT, Phys. Lett. B 442, 109 (1998).

[7] E. G. Gimon and C. Popescu, The operator spectrum of the six-dimensional $(1,0)$ theory, J. High Energy Phys. 04 (1999) 018.

[8] F. Apruzzi, M. Fazzi, D. Rosa, and A. Tomasiello, All AdS solutions of type II supergravity, J. High Energy Phys. 04 (2014) 064.

[9] D. Gaiotto and A. Tomasiello, Holography for $(1,0)$ theories in six dimensions, J. High Energy Phys. 12 (2014) 003.

[10] A. Passias, A. Rota, and A. Tomasiello, Universal consistent truncation for $6 \mathrm{~d} / 7 \mathrm{~d}$ gauge/gravity duals, J. High Energy Phys. 10 (2015) 187.
[11] F. Apruzzi, G. Dibitetto, and L. Tizzano, A new 6d fixed point from holography, J. High Energy Phys. 11 (2016) 126.

[12] F. Apruzzi and M. Fazzi, $\mathrm{AdS}_{7} / \mathrm{CFT}_{6}$ with orientifolds, J. High Energy Phys. 01 (2018) 124.

[13] E. Bergshoeff, I. G. Koh, and E. Sezgin, Yang-MillsEinstein supergravity in seven dimensions, Phys. Rev. D 32, 1353 (1985).

[14] Y. J. Park, Gauged Yang-Mills-Einstein supergravity with three index field in seven dimensions, Phys. Rev. D 38, 1087 (1988).

[15] A. Salam and E. Sezgin, $S O(4)$ gauging of $N=2$ supergravity in seven-dimensions, Phys. Lett. 126B, 295 (1983).

[16] P. K. Townsend and P. van Nieuwenhuizen, Gauged sevendimensional supergravity, Phys. Lett. 125B, 41 (1983).

[17] L. Mezincescu, P. K. Townsend, and P. van Nieuwenhuizen, Stability of gauged $d=7$ supergravity and the definition of masslessness in $\mathrm{AdS}_{7}$, Phys. Lett. 143B, 384 (1984).

[18] E. Bergshoeff, D. C. Jong, and E. Sezgin, Noncompact gaugings, chiral reduction and dual sigma model in supergravity, Classical Quantum Gravity 23, 2803 (2006).

[19] P. Karndumri, RG flows in 6D $N=(1,0)$ SCFT from $S O(4)$ half-maximal gauged supergravity, J. High Energy Phys. 06 (2014) 101.

[20] P. Karndumri, Noncompact gauging of $N=2$ 7D supergravity and AdS/CFT holography, J. High Energy Phys. 02 (2015) 034.

[21] P. Karndumri, RG flows from $(1,0) 6 \mathrm{D}$ SCFTs to $N=1$ SCFTs in four and three dimensions, J. High Energy Phys. 06 (2015) 027. 
[22] J. Louis and S. Lüst, Supersymmetric $\operatorname{AdS}_{7}$ backgrounds in half-maximal supergravity and marginal operators of $(1,0)$ SCFTs, J. High Energy Phys. 10 (2015) 120.

[23] G. Dibitetto and N. Petri, BPS objects in $D=7$ supergravity and their M-theory origin, J. High Energy Phys. 12 (2017) 041.

[24] H. Lu and C. N. Pope, Exact embedding of $N=1, D=7$ gauged supergravity in $D=11$, Phys. Lett. B 467, 67 (1999).

[25] G. Dibitetto and N. Petri, 6d surface defects from massive type IIA, J. High Energy Phys. 01 (2018) 039.
[26] G. Dibitetto, J. J. Fernndez-Melgarejo, and D. Marqus, All gaugings and stable de Sitter in $D=7$ half-maximal supergravity, J. High Energy Phys. 11 (2015) 037.

[27] P. Karndumri, $N=2 S O(4) 7 \mathrm{D}$ gauged supergravity with topological mass term from 11 dimensions, J. High Energy Phys. 11 (2014) 063.

[28] S. S. Gubser, Curvature singularities: The good, the bad and the naked, Adv. Theor. Math. Phys. 4, 679 (2000).

[29] J. Maldacena and C. Nunez, Supergravity description of field theories on curved manifolds and a no go theorem, Int. J. Mod. Phys. A 16, 822 (2001). 